\title{
Assessment of Undiscovered Oil and Gas Resources in Tertiary Strata of the Gulf Coast, 2007
}

$U_{\text {sing a geology-based assessment methodology, }}$ the U.S. Geological Survey estimated a mean of 113.7 trillion cubic feet of undiscovered natural gas, a mean of 690 million barrels of undiscovered oil, and a mean of 3.7 billion barrels of undiscovered natural gas liquids in onshore lands and State waters of the Gulf Coast.

\section{Introduction}

The U.S. Geological Survey (USGS) recently completed an assessment of the undiscovered oil and gas resources in Tertiary strata and coalbed gas resources in Cretaceous-Tertiary strata of the onshore areas and State waters of the Gulf Coast (fig. 1). The assessment is based on geologic elements of a total petroleum system (TPS), including characterization of hydrocarbon source rocks (source-rock maturation, hydrocarbon generation and migration), reservoir rocks (sequence stratigraphy and petrophysical properties), and hydrocarbon traps (trap formation, timing, and seals). Using these criteria, the USGS defined an Upper Jurassic-Cretaceous-Tertiary Composite TPS for conventional oil and gas resources that extends around the entire Gulf of Mexico, including portions of both the United States and Mexico. However, the present assessment of undiscovered conventional oil and gas resources includes only that portion of the TPS that lies onshore and in State waters of the United States (fig. 1). In addition, the USGS defined three self-sourced coalbed gas TPSs. Two are entirely

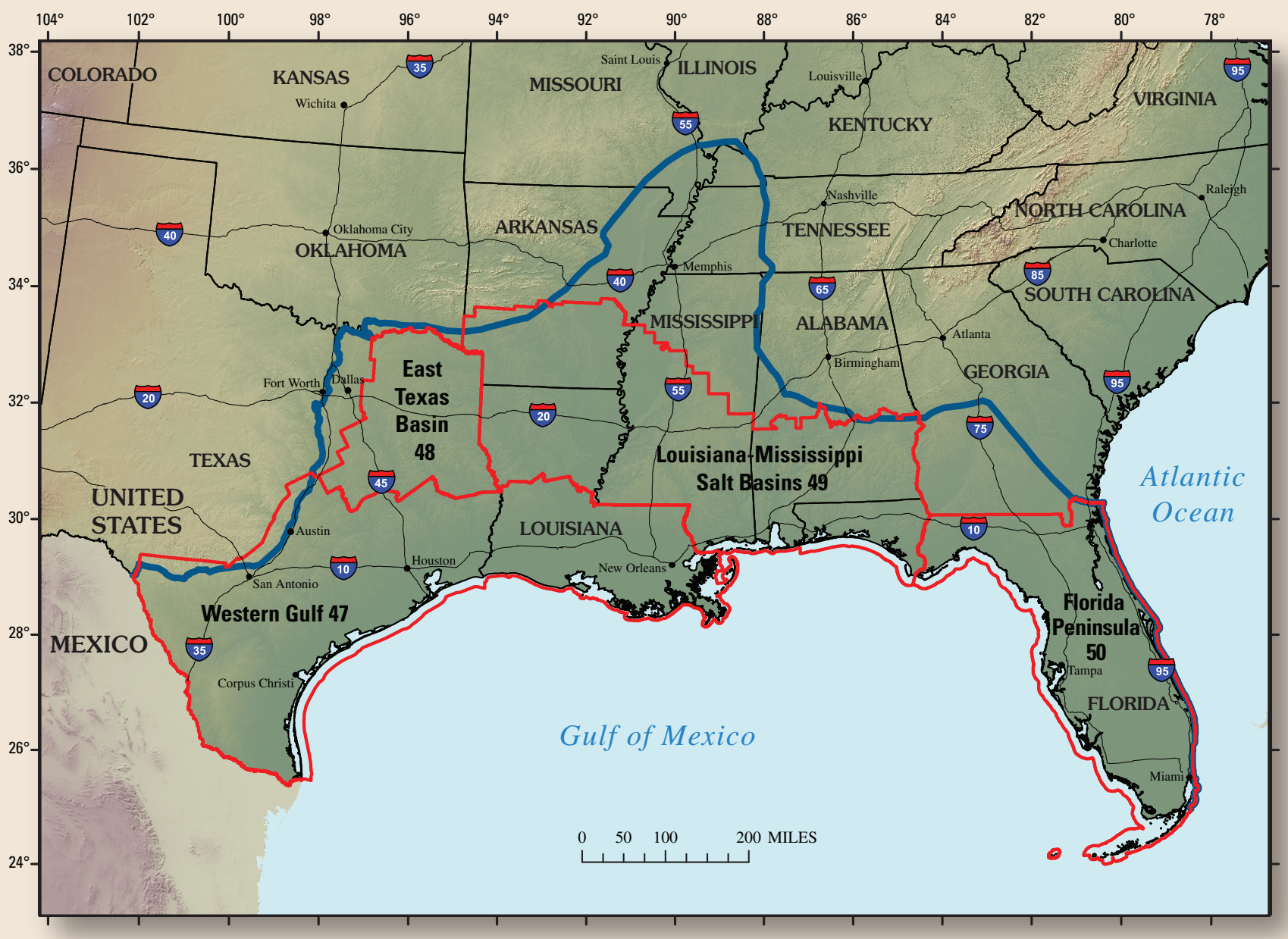

Figure 1. Map showing the part of the Upper Jurassic-Cretaceous-Tertiary Composite Total Petroleum System that is onshore and in State waters of the Gulf Coast of the United States (blue line). Province boundaries defined by the U.S. Geological Survey are indicated by red outlines. 
Table 1. Gulf Coast assessment results.

[MMBO, million barrels of oil. BCFG, billion cubic feet of gas. MMBNGL, million barrels of natural gas liquids. Results shown are fully risked estimates. For gas accumulations, all liquids are included as NGL (natural gas liquids). F95 represents a 95 percent chance of at least the amount tabulated; other fractiles are defined similarly. Fractiles are additive under the assumption of perfect positive correlation. TPS, total petroleum system; AU, assessment unit. Gray shading indicates not applicable]

\begin{tabular}{|c|c|c|c|c|c|c|c|c|c|c|c|c|c|}
\hline \multirow{3}{*}{$\begin{array}{l}\text { Total Petroleum Systems } \\
\text { (TPS) } \\
\text { and Assessment Units (AU) }\end{array}$} & \multirow{3}{*}{$\begin{array}{l}\text { Field } \\
\text { Type }\end{array}$} & \multicolumn{12}{|c|}{ Total Undiscovered Resources } \\
\hline & & \multicolumn{4}{|c|}{ Oil (MMBO) } & \multicolumn{4}{|c|}{ Gas (BCFG) } & \multicolumn{4}{|c|}{ NGL (MMBNGL) } \\
\hline & & F95 & F50 & F5 & Mean & F95 & F50 & F5 & Mean & F95 & F50 & F5 & Mean \\
\hline \multicolumn{14}{|c|}{ Upper Jurassic-Cretaceous-Tertiary Composite TPS (504701) } \\
\hline \multirow{2}{*}{$\begin{array}{l}\text { Wilcox Stable Shelf Oil } \\
\text { and Gas AU (50470116) }\end{array}$} & Oil & 12 & 49 & 111 & 54 & 8 & 33 & 83 & 38 & 0 & 1 & 3 & 1 \\
\hline & Gas & & & & & 117 & 403 & 846 & 434 & 4 & 13 & 30 & 14 \\
\hline \multirow{2}{*}{$\begin{array}{l}\text { Wilcox Expanded Fault Zone } \\
\text { Gas and Oil AU (50470117) }\end{array}$} & Oil & 18 & 49 & 95 & 52 & 74 & 215 & 459 & 234 & 2 & 6 & 14 & 7 \\
\hline & Gas & & & & & 714 & 2,114 & 4,299 & 2,264 & 20 & 61 & 136 & 68 \\
\hline \multirow{2}{*}{$\begin{array}{l}\text { Wilcox Slope and Basin Floor } \\
\text { Gas AU (50470118) }\end{array}$} & Oil & 0 & 0 & 0 & 0 & 0 & 0 & 0 & 0 & 0 & 0 & 0 & 0 \\
\hline & Gas & & & & & 5,173 & 23,629 & 56,486 & 26,398 & 78 & 366 & 959 & 423 \\
\hline \multirow{2}{*}{$\begin{array}{l}\text { Wilcox-Lobo Slide Block Gas } \\
\text { AU (50470119) }\end{array}$} & Oil & 1 & 4 & 9 & 4 & 6 & 20 & 48 & 23 & 0 & 0 & 1 & 1 \\
\hline & Gas & & & & & 1,543 & 6,803 & 15,732 & 7,498 & 24 & 109 & 276 & 125 \\
\hline \multirow{2}{*}{$\begin{array}{l}\text { Lower Claiborne Stable Shelf } \\
\text { Gas and Oil AU (50470120) }\end{array}$} & Oil & 3 & 7 & 13 & 7 & 7 & 19 & 40 & 21 & 0 & 0 & 1 & 0 \\
\hline & Gas & & & & & 21 & 63 & 124 & 67 & 0 & 2 & 3 & 2 \\
\hline \multirow{2}{*}{$\begin{array}{l}\text { Lower Claiborne Expanded } \\
\text { Fault Zone Gas AU (50470121) }\end{array}$} & Oil & 1 & 3 & 8 & 4 & 6 & 16 & 43 & 19 & 0 & 1 & 3 & 1 \\
\hline & Gas & & & & & 351 & 942 & 1,767 & 987 & 13 & 37 & 76 & 39 \\
\hline \multirow{2}{*}{$\begin{array}{l}\text { Lower Claiborne Slope and } \\
\text { Basin Floor Gas AU (50470122) }\end{array}$} & Oil & 0 & 0 & 0 & 0 & 0 & 0 & 0 & 0 & 0 & 0 & 0 & 0 \\
\hline & Gas & & & & & 573 & 3,195 & 8,044 & 3,620 & 21 & 124 & 338 & 145 \\
\hline \multirow{2}{*}{$\begin{array}{l}\text { Lower Claiborne Cane River } \\
\text { AU (50470123) }\end{array}$} & Oil & \multirow{2}{*}{\multicolumn{12}{|c|}{ Not quantitatively assessed }} \\
\hline & Gas & & & & & & & & & & & & \\
\hline \multirow{2}{*}{$\begin{array}{l}\text { Upper Claiborne Stable Shelf } \\
\text { Gas and Oil AU (50470124) }\end{array}$} & Oil & 4 & 12 & 23 & 13 & 12 & 36 & 78 & 40 & 0 & 1 & 2 & 1 \\
\hline & Gas & & & & & 97 & 357 & 858 & 402 & 3 & 10 & 25 & 11 \\
\hline \multirow{2}{*}{$\begin{array}{l}\text { Upper Claiborne Expanded } \\
\text { Fault Zone Gas AU (50470125) }\end{array}$} & Oil & 8 & 26 & 53 & 28 & 39 & 128 & 287 & 142 & 3 & 9 & 21 & 10 \\
\hline & Gas & & & & & 1,417 & 4,386 & 9,096 & 4,740 & 95 & 307 & 698 & 341 \\
\hline \multirow{2}{*}{$\begin{array}{l}\text { Upper Claiborne Slope and } \\
\text { Basin Floor Gas AU (50470126) }\end{array}$} & Oil & 0 & 0 & 0 & 0 & 0 & 0 & 0 & 0 & 0 & 0 & 0 & 0 \\
\hline & Gas & & & & & 1,706 & 8,147 & 19,632 & 9,107 & 116 & 569 & 1,489 & 655 \\
\hline Jackson Stable Shelf Oil and & Oil & 2 & 4 & 10 & 5 & 2 & 6 & 16 & 7 & 0 & 0 & 0 & 0 \\
\hline Gas AU (50470127) & Gas & & & & & 7 & 16 & 32 & 17 & 0 & 0 & 1 & 1 \\
\hline Jackson Expanded Fault Zone & Oil & 5 & 17 & 40 & 19 & 7 & 26 & 66 & 30 & 0 & 1 & 3 & 1 \\
\hline Gas and Oil AU (50470128) & Gas & & & & & 110 & 449 & 1,036 & 495 & 4 & 16 & 39 & 18 \\
\hline Jackson Slope and Basin Floor & Oil & 0 & 0 & 0 & 0 & 0 & 0 & 0 & 0 & 0 & 0 & 0 & 0 \\
\hline Gas AU (50470129) & Gas & & & & & 94 & 393 & 929 & 438 & 3 & 15 & 39 & 18 \\
\hline Jackson Louisiana-Mississippi- & Oil & Net & & & & & & & & & & & \\
\hline Alabama Area AU (50470130) & Gas & I & 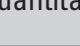 & ely a & essec & & & & & & & & \\
\hline Vicksburg Stable Shelf Oil and & Oil & 5 & 16 & 35 & 17 & 18 & 61 & 147 & 69 & 0 & 1 & 4 & 2 \\
\hline Gas AU (50470131) & Gas & & & & & 39 & 114 & 218 & 120 & 1 & 3 & 5 & 3 \\
\hline Vicksburg Expanded Fault Zone & Oil & 6 & 19 & 41 & 21 & 17 & 58 & 133 & 65 & 1 & 3 & 7 & 3 \\
\hline Gas and Oil AU (50470132) & Gas & & & & & 2,057 & 8,598 & 19,880 & 9,511 & 68 & 295 & 747 & 336 \\
\hline Vicksburg Slope and Basin & Oil & 0 & 0 & 0 & 0 & 0 & 0 & 0 & 0 & 0 & 0 & 0 & 0 \\
\hline Floor Gas AU (50470133) & Gas & & & & & 1,607 & 6,920 & 15,840 & 7,621 & 61 & 269 & 668 & 305 \\
\hline Vicksburg Mississippi-Alabama & Oil & Not &. & 1 & ssesseo & & & & & & & & \\
\hline Area AU (50470134) & Gas & & & & & & & & & & & & \\
\hline Frio Stable Shelf Oil and Gas & Oil & 2 & 5 & 11 & 5 & 7 & 22 & 52 & 25 & 0 & 0 & 1 & 0 \\
\hline AU (50470135) & Gas & & & & & 88 & 227 & 437 & 241 & 2 & 5 & 11 & 6 \\
\hline Frio Expanded Fault Zone Oil & Oil & 4 & 14 & 30 & 16 & 24 & 82 & 186 & 90 & 1 & 2 & 5 & 2 \\
\hline and Gas AU (50470136) & Gas & & & & & 509 & 1,265 & 2,292 & 1,321 & 13 & 34 & 68 & 36 \\
\hline
\end{tabular}


Table 1. Gulf Coast assessment results._Continued

\begin{tabular}{|c|c|c|c|c|c|c|c|c|c|c|c|c|c|}
\hline \multirow{3}{*}{$\begin{array}{l}\text { Total Petroleum Systems } \\
\text { (TPS) } \\
\text { and Assessment Units (AU) }\end{array}$} & \multirow{3}{*}{$\begin{array}{l}\text { Field } \\
\text { Type }\end{array}$} & \multicolumn{12}{|c|}{ Total Undiscovered Resources } \\
\hline & & \multicolumn{4}{|c|}{ Oil (MMBO) } & \multicolumn{4}{|c|}{ Gas (BCFG) } & \multicolumn{4}{|c|}{ NGL (MMBNGL) } \\
\hline & & F95 & F50 & F5 & Mean & F95 & F50 & F5 & Mean & F95 & F50 & F5 & Mean \\
\hline \multicolumn{14}{|c|}{ Upper Jurassic-Cretaceous-Tertiary Composite TPS (504701)—Continued } \\
\hline \multirow{2}{*}{$\begin{array}{l}\text { Frio Slope and Basin Floor Gas } \\
\text { AU (50470137) }\end{array}$} & Oil & 28 & 102 & 220 & 110 & 84 & 320 & 756 & 358 & 2 & 9 & 24 & 11 \\
\hline & Gas & & & & & 1,271 & 4,829 & 10,397 & 5,231 & 81 & 322 & 757 & 358 \\
\hline \multirow{2}{*}{$\begin{array}{l}\text { Anahuac Oil and Gas } \\
\text { AU (50470138) }\end{array}$} & Oil & 3 & 13 & 39 & 16 & 8 & 33 & 103 & 41 & 0 & 1 & 2 & 1 \\
\hline & Gas & & & & & 62 & 240 & 578 & 270 & 2 & 7 & 17 & 8 \\
\hline \multirow{2}{*}{$\begin{array}{l}\text { Hackberry Oil and Gas } \\
\text { AU (50470139) }\end{array}$} & Oil & 6 & 22 & 52 & 25 & 17 & 69 & 178 & 80 & 0 & 2 & 6 & 2 \\
\hline & Gas & & & & & 539 & 1,632 & 3,187 & 1,727 & 34 & 109 & 233 & 118 \\
\hline \multirow{2}{*}{$\begin{array}{l}\text { Lower Miocene Shelf Oil and } \\
\text { Gas AU (50470140) }\end{array}$} & Oil & 4 & 15 & 40 & 17 & 9 & 34 & 99 & 42 & 0 & 0 & 1 & 1 \\
\hline & Gas & & & & & 82 & 301 & 641 & 324 & 1 & 5 & 11 & 5 \\
\hline \multirow{2}{*}{$\begin{array}{l}\text { Lower Miocene Slope and } \\
\text { Basin Gas AU (50470141) }\end{array}$} & Oil & 5 & 16 & 37 & 18 & 20 & 74 & 181 & 84 & 1 & 2 & 6 & 3 \\
\hline & Gas & & & & & 5,786 & 17,582 & 37,327 & 19,116 & 137 & 445 & 1,027 & 496 \\
\hline \multirow{2}{*}{$\begin{array}{l}\text { Middle Miocene Shelf Oil and } \\
\text { Gas AU (50470142) }\end{array}$} & Oil & 5 & 21 & 56 & 25 & 21 & 92 & 263 & 110 & 0 & 2 & 6 & 2 \\
\hline & Gas & & & & & 673 & 3,000 & 7,040 & 3,321 & 12 & 54 & 140 & 62 \\
\hline \multirow{2}{*}{$\begin{array}{l}\text { Middle Miocene Slope and } \\
\text { Basin Gas AU (50470143) }\end{array}$} & Oil & 25 & 115 & 315 & 135 & 75 & 358 & 1,040 & 432 & 2 & 8 & 26 & 10 \\
\hline & Gas & & & & & 277 & 1,242 & 3,081 & 1,405 & 7 & 30 & 81 & 35 \\
\hline \multirow{2}{*}{$\begin{array}{l}\text { Upper Miocene Shelf Oil and } \\
\text { Gas AU (50470144) }\end{array}$} & Oil & 7 & 28 & 70 & 32 & 9 & 40 & 108 & 47 & 0 & 1 & 1 & 1 \\
\hline & Gas & & & & & 101 & 441 & 1,077 & 498 & 3 & 13 & 33 & 15 \\
\hline \multirow{2}{*}{$\begin{array}{l}\text { Upper Miocene Slope and } \\
\text { Basin Gas AU (50470145) }\end{array}$} & Oil & 8 & 33 & 88 & 38 & 40 & 168 & 483 & 203 & 1 & 5 & 16 & 6 \\
\hline & Gas & & & & & 41 & 144 & 320 & 158 & 2 & 8 & 20 & 9 \\
\hline \multirow{2}{*}{$\begin{array}{l}\text { Plio-Pleistocene Shelf Oil and } \\
\text { Gas AU (50470146) }\end{array}$} & Oil & 7 & 26 & 62 & 29 & 9 & 37 & 95 & 43 & 0 & 0 & 1 & 1 \\
\hline & Gas & & & & & 21 & 70 & 154 & 77 & 1 & 4 & 10 & 5 \\
\hline \multirow{2}{*}{$\begin{array}{l}\text { Wilcox Mississippi Embayment } \\
\text { AU (50470147) }\end{array}$} & Oil & \multirow{2}{*}{\multicolumn{12}{|c|}{ Not quantitatively assessed }} \\
\hline & Gas & & & & & & & & & & & & \\
\hline \multirow{2}{*}{$\begin{array}{l}\text { Frio Basin Margin } \\
\mathrm{AU}(50470148)\end{array}$} & Oil & \multirow{2}{*}{\multicolumn{12}{|c|}{ Not quantitatively assessed }} \\
\hline & Gas & & & & & & & & & & & & \\
\hline $\begin{array}{l}\text { Total Conventional } \\
\text { Resources }\end{array}$ & & 169 & 616 & 1,458 & 690 & 25,595 & 99,449 & 226,294 & 109,651 & 819 & 3,287 & 8,091 & 3,724 \\
\hline \multicolumn{14}{|l|}{ OImos Coalbed Gas TPS (504702) } \\
\hline $\begin{array}{l}\text { Cretaceous Olmos Coalbed Gas } \\
\text { AU (50470281) }\end{array}$ & $C B G$ & & & & & 37 & 70 & 133 & 75 & 0 & 0 & 1 & 0 \\
\hline $\begin{array}{l}\text { Rio Escondido Basin Olmos } \\
\text { Coalbed Gas AU (53000281) }\end{array}$ & $C B G$ & & & & & 60 & 114 & 215 & 123 & 0 & 0 & 1 & 0 \\
\hline \multicolumn{14}{|l|}{ Wilcox Coalbed Gas TPS (504703) } \\
\hline $\begin{array}{l}\text { Wilcox Coalbed Gas } \\
\text { AU (50470381) }\end{array}$ & $C B G$ & & & & & 1,565 & 3,442 & 7,570 & 3,861 & 0 & 0 & 0 & 0 \\
\hline Cretaceous-Tertiary Coalbed Ga & s TPS 1 & 304704) & & & & & & & & & & & \\
\hline $\begin{array}{l}\text { Cretaceous-Tertiary Coalbed } \\
\text { Gas AU (50470481) }\end{array}$ & CBG & Not qu & antitati & ely ass & essed & & & & & & & & \\
\hline $\begin{array}{l}\text { Total Continuous } \\
\text { Resources }\end{array}$ & & & & & & 1,662 & 3,626 & 7,918 & 4,059 & 0 & $\mathbf{0}$ & 2 & 0 \\
\hline $\begin{array}{l}\text { Total Undiscovered } \\
\text { Oil and Gas Resources }\end{array}$ & & 169 & 616 & 1,458 & 690 & 27,257 & 103,075 & 234,212 & 113,710 & 819 & 3,287 & 8,093 & 3,724 \\
\hline
\end{tabular}


within the United States, and the third has one assessment unit (AU) in the United States and one AU in Mexico. The portion of the composite conventional TPS that lies within the United States covers a geographic area that extends from the southwest boundary of the Western Gulf Province on the Texas-Mexico border eastward into Texas and Louisiana, including the East Texas Basin Province, and continues to State waters and onshore lands primarily in Louisiana, Mississippi, Arkansas, and Alabama in the Louisiana-Mississippi Salt Basins Province. The TPS also includes Florida and small parts of Oklahoma, Missouri, Illinois, Kentucky, Tennessee, and Georgia (fig. 1). The Florida Peninsula Province was not assessed as part of this study. The Upper Jurassic-Cretaceous-Tertiary Composite TPS contains 33 AUs for undiscovered conventional oil and (or) gas resources (table 1) that lie predominantly on the coastal plain and in State waters of Texas, Louisiana, Mississippi, Alabama, and a small part of the Florida panhandle. The three coalbed TPSs contain four AUs (table 1).

\section{Resource Summary}

The USGS assessment of undiscovered conventional oil and gas resources and continuous coalbed gas resources resulted in estimated means of 113.7 trillion cubic feet of gas (TCFG), 690 million barrels of oil (MMBO), and 3.7 billion barrels of natural gas liquids in the AUs that were assessed (table 1). A large portion of the undiscovered gas resource is considered to be in conventional accumulations trapped in sedimentary strata ranging in age from Paleocene to Pliocene-Pleistocene (fig. 2). The remaining undiscovered gas resource occurs as continuous coalbed gas in Tertiary and Cretaceous-Tertiary strata. The conventional oil and gas resources reside primarily in clastic reservoirs deposited on continental shelves, in shelf-margin deltas, and in slope fans and basin-floor fans. The continuous coalbed gas resources are present within coal beds.

\section{For Additional Information}

Supporting geologic studies of Gulf Coast region total petroleum systems and assessment units are in progress, as well as studies of the methodology used in the assessment of both conventional resources in Tertiary strata and continuous coalbed gas resources in Cretaceous-Tertiary rocks of the Gulf Coast. Assessment results are available at the USGS Central Energy Team web site: http://energy.cr.usgs.gov/oilgas/noga

\section{Gulf Coast Assessment Team}

Neogene: Russell F. Dubiel (Task Leader, rdubiel@usgs.gov), Janet K. Pitman, Ofori N. Pearson

Paleogene: Peter D. Warwick (Task Leader, pwarwick@usgs.gov), Alexander W. Karlsen, James L. Coleman, Paul C. Hackley, Daniel O. Hayba, Sharon M. Swanson

Review Committee: Ronald R. Charpentier, Troy A. Cook, Timothy R. Klett, Richard M. Pollastro, Christopher J. Schenk

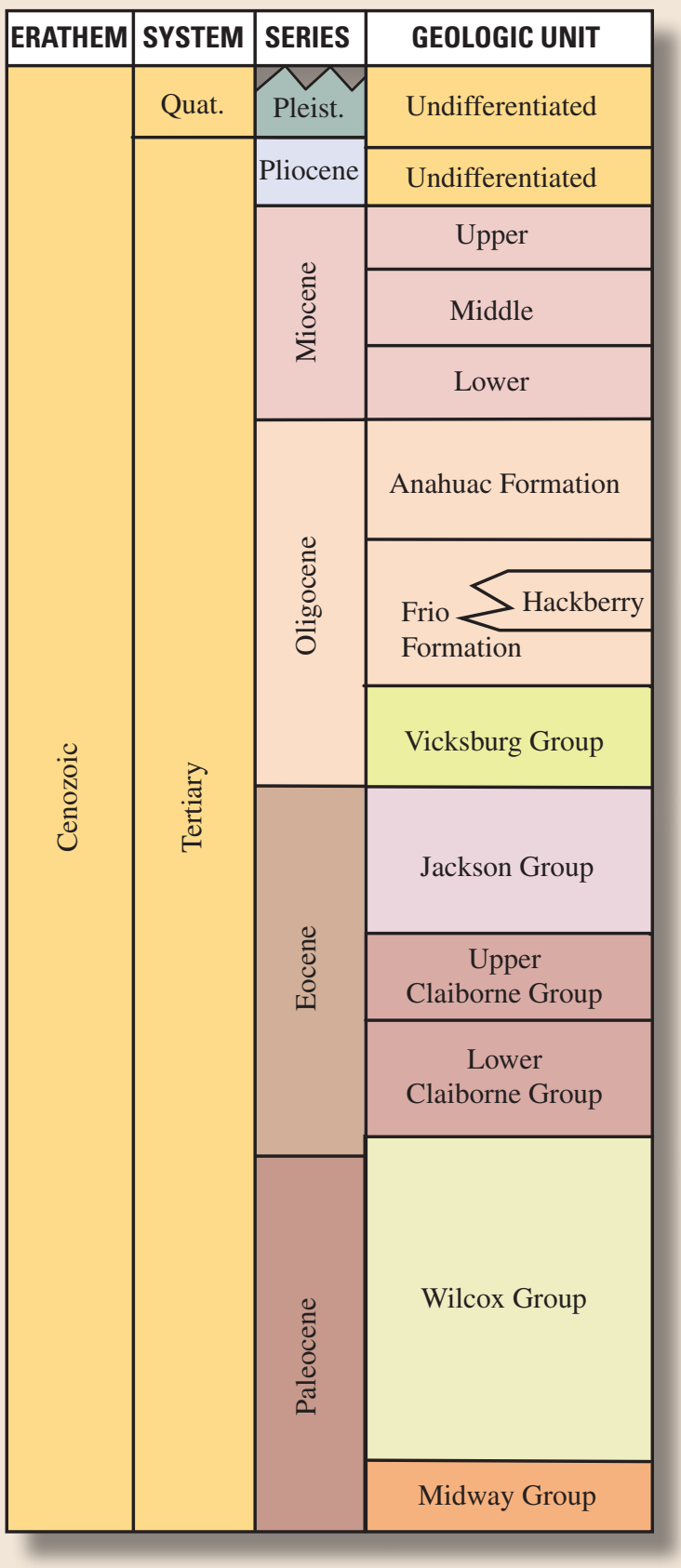

Figure 2. Generalized stratigraphic section showing geologic units that were assessed in the Gulf Coast region. Nomenclature is a combination of formal and informal groups, and formation and member names that are based on physical or biostratigraphic correlations in outcrop and subsurface studies. The nomenclature reflects the common designation and usage in the region by State, industry, and academic geologists. 